\title{
Management strategies for run-of-river hydropower plants: an optimal switching approach
}

\author{
Marcus Olofsson ${ }^{1} \cdot$ Thomas Önskog $^{2} \cdot$ Niklas L. P. Lundström $^{1}$
}

Received: 7 May 2021 / Revised: 19 August 2021 / Accepted: 19 August 2021 /

Published online: 5 September 2021

(c) The Author(s) 2021

\begin{abstract}
The mathematical theory for optimal switching is by now relatively well developed, but the number of concrete applications of this theoretical framework remains few. In this paper, we bridge parts of this gap by applying optimal switching theory to a conceptual production planning problem related to hydropower. In particular, we study two examples of small run-of-river hydropower plants and provide an outline of how optimal switching can be used to create fully automatic production schemes for these. Along the way, we create a new model for random flow of water based on stochastic differential equations and fit this model to historical data. We benchmark the performance of our model using actual flow data from a small river in Sweden and find that our production scheme lies close to the optimal, within 2 and $5 \%$, respectively, in a long term investigation of the two plants considered.
\end{abstract}

Keywords Stochastic differential equation · River flow model $\cdot$ Partial differential equation $\cdot$ Variational inequality $\cdot$ Production planning $\cdot$ Optimization

Mathematics Subject Classification 35K45 - 49N90 - 90B50

Marcus Olofsson

marcus.olofsson@math.umu.se

Thomas Önskog

thomas.onskog@scb.se

Niklas L. P. Lundström

niklas.lundstrom@umu.se

1 Department of Mathematics and Mathematical Statistics, Umeå University, 90187 Umeå, Sweden

2 Statistics Sweden, Stockholm 171 54, Solna, Sweden 


\section{Introduction}

Small-scale hydropower plants are in many cases of "run-of-river" type (ROR) meaning that any dam or barrage is small, usually just a weir, and generally little or no water can be stored. ROR hydropower plants preserve the natural flow of the river (besides of course at the location of the power plant) and are therefore among the most environmentally benign existing energy technologies. Due to a low installation cost, ROR hydropower plants are often cost-efficient and, as such, useful both for rural electrification in developing countries and for new hydropower developments in industrialized countries (Paish 2002). ROR hydropower plants are common in smaller rivers but also exist in larger sizes such as the Niagara Falls hydroelectric plants (Canada/USA), the Chief Joseph dam on the Columbia River (Washington, USA) or the Saint Marys Falls hydropower plant in Sault Sainte Marie (Michigan, USA).

The optimal sizing of ROR hydropower plants has been considered by several authors, see, e.g., (Anagnostopoulos and Papantonis 2007; Bozorg Haddad et al. 2011; Fahlbusch 1983; Garrido et al. 2009) and the references therein. In the estimation of the performance of a given power plant, all these authors omit the cost of switching between different production modes. Doing so, the optimal management strategy can be found trivially by starting the machine when flow is sufficient and stopping it when flow is insufficient. However, in rivers with large and rapid flow fluctuations, which is typically the case in smaller unregulated rivers, such strategies can lead to a large number of changes in the production, the cost of which can not be neglected; for example, starting and stopping the turbines induces wear and tear on the machines and may also require intervention from personnel. Moreover, each start and stop involves a risk which can be considered a cost. To give an example, the major breakdown in the Akkats hydropower plant (Lule river, Sweden) 2002 was caused by a turbine being stopped too quickly, resulting in rushing water destroying the foundation of both the turbine and the generator (Yang 2010; Yang et al. 2018).

In this paper we create fully automatic production schemes for two canonical examples of ROR hydropower plants, with stochastic flow of water and with nonzero cost of switching between different states of production. Our method is based on optimal switching and provides, to the best of our knowledge, a novel way to handle hydropower production planning using stochastic control theory. Along the way of deriving the production strategies, we create a new model for river flow based on stochastic differential equations (SDEs) and fit this model to historical data. The stochastic flow model can incorporate a given flow forecast in order to catch up short term fluctuations.

Our primary goal is to provide the reader with a theroretical background of optimal switching theory and to give an accessible walk-through of the steps required to apply the theory to real-life production planning. We therefore stay within a simple setup, studying management of a single (fictitious) power plant without storage capacity or means to control the flow of water, i.e., a ROR hydropower plant, with few different production states and a single underlying stochastic quantity (the flow 
of water) affecting the production. Although this is restrictive from a practitioner's point of view, we stress that the mathematical framework presented here can be extended to multiple production states and multiple underlying stochastic processes influencing the production; the cost of such generalizations being mainly computational. We also expect the theory to be useful in much more general settings, including hydropower plants with dams and sequential power plants.

The rest of this paper is outlined as follows. In Sect. 1.1 we give a literature survey and explain the contribution of this paper. Section 2 contains an introduction to optimal switching problems and, in particular, an outline of the theory in the context of hydropower planning. (The mathematics presented is well-known in the stochastic control-community, but it is nevertheless non-trivial and we believe the more applications-minded reader may benefit from an overview.) Sections 3 and 4 contain models for the flow of water and the power plants under consideration, respectively. Section 5 contains a very brief outline of the numerical approach taken to solve the variational inequalities appearing. We present the result of our parameter estimation and the performance of the constructed strategy in Sect. 6 and end with Sect. 7 in which we discuss our approach in general, and our results in particular, and make some concluding remarks.

\subsection{Literature survey and our contribution}

Optimal switching is a relatively new and fast growing field of mathematics combining optimization, SDEs and partial differential equations (PDEs) (Barkhudaryan et al. 2020; Biswas et al. 2010; Djehiche et al. 2010; El-Asri and Fakhouri 2017; El-Asri and Hamadéne 2009; Hamadéne and Morlais 2013; Hu and Tang 2010; Kharroubi 2016; Lundström et al. 2014a, b, 2019; Martyr 2016a, b; Perninge 2018, 2020, 2020, Lundström and Olofsson 2021). However, a literature survey shows that, although the mathematical theory is well developed, applications of optimal switching to real life problems is a far less explored area. A possible explanation for this discrepancy could be the difficulty of formulating real problems in mathematical terms and, conversely, to interpret the theoretical results in practical terms.

Most commonly, applications are found in the context of real options, see (Brekke and Øksendal 1994; Brennan and Schwartz 1985; Carmona and Ludkovski 2008; Duckworth and Zervos 2001; Goutte et al. 2018). In Aïd et al. (2014) the authors provide a probabilistic numerical scheme for optimal switching problems and test their scheme on a fictitious problem of investment in electricity generation. In Carmona and Ludkovski (2010) valuation of energy storage is studied with the help of optimal switching and in Perninge (2020), Perninge and Eriksson (2017) the authors study how the framework can be used to track electricity demand.

A main feature of optimal switching-based production planning is that it allows for random factors influencing the production strategy (for details see Sect. 2 below). This randomness is in general given by a Markovian stochastic process and can incorporate any number of different variables. However, to reduce computational strain we focus on how the flow of water impacts the production. Popular streamflow models include linear time series models, such as ARMA models with Gaussian or 
GARCH noise (Modarres and Ouarda 2013; Mujumdar and Nagesh Kumar 1990; Wang et al. 2005), and non-linear time series models, such as SETAR models (Fathian et al. 2019). Modern approaches also include neural networks and machine learning techniques, see, e.g., (Moeeni et al. 2017; Matos and Schleiss 2017) and the references therein. Another suitable approach, and the approach that we have adopted here due to its natural relation to the optimal switching framework, is SDEs driven by Gaussian white noise and/or compound Poissonian impulses (Bodo et al. 1987). In particular, we develop a new stand-alone SDE-based model for the flow of water $Q$, based on historical data and driven by Brownian motion, which mimics the long term seasonal variations of the flow while still allowing for short term fluctuations and flow forecasts.

When trying to maximize monetary profit instead of $k W h$ of produced electricity, the electricity spot price $P_{t}$ at which the electricity produced at $t$ is sold is of course also of interest when planning the production. In general, $P$ is an exogenous stochastic process which can be incorporated into our model similarly to the flow of water. However, modelling electricity prices is a non-trivial task and prices are usually not Itô diffusions, but rather discontinuous jump-processes, see, e.g., (Hellström et al. 2012; Weron et al. 2004, 2004), making the operator in the variational inequality to be solved non-local (see, e.g., Lundström et al. 2014a, b). For simplicity, we therefore let $P$ be a continuous deterministic process in the current paper. We stress, however, that our approach can readily be extended to random electricity prices (and streamflow models) with compound Poisson impulses at the cost of computational complexity.

This paper extends the use of optimal switching theory by applying the general framework to two canonical examples of ROR hydropower plants. In particular, we adapt and calibrate the theory to construct management strategies which we benchmark using real flow data. We deliberately work in a rather simplistic setting, using fictitious power plants and general parameters. Our contribution should be seen as conceptual; we outline the steps required to apply the optimal switching theory in practice and indicate with numerical examples that the constructed strategies are close to optimal. Adaptation for use in any particular case must be done "on-site" to account for the specific conditions.

We have chosen not to let deeper knowledge of different types of generators and how they operate be a prerequisite for the understanding of this paper and we hence avoid going into any such technical details. A comprehensive survey of different turbines and generators and their distinct characteristics can be found in, e.g., (Boldea 2015; Brekke 2001; Warnick 1984).

\section{Optimal switching in the context of hydropower}

Tailored to the setting of hydropower plants, the optimal switching problem can be described as follows: We consider a manager of a hydropower plant with several units, each unit being a sub-power plant, i.e., a turbine and a generator. Each unit can be started and stopped separately in order to adjust the production of the power 
plant to the supply of water or to the production demand. This implies that the manager has the option to run the plant in $m \geq 2$ production modes, corresponding to running different combinations of units. Starting and stopping units induces wear and tear on the units and therefore the manager finds herself in a trade-off, weighing the benefits of changing production mode/state against the costs induced by making these changes.

Let $X=\left\{X_{t}\right\}_{t \geq 0}$ denote a Markovian stochastic process representing the features which influence the production. For small hydropower plants, $X$ may represent the flow of the river, but it may also be interpreted as, e.g., production demand for a frequency regulating plant, the spot price of electricity, or a varying cost of production such as for an oil driven power plant. The process $X$ may be multi-dimensional and hence incorporate all of the above and more (but for simplicity we will in the bulk of this paper consider $X=(Q, P)$ where $Q$ and $P$ are one-dimensional processes representing flow of water and deterministic price of electricity, respectively). Moreover, we let $f_{i}\left(X_{t}, t\right)$ denote the instantaneous payoff generated in production mode $i$ at time $t$, when the state of the underlying process is $X_{t}$. Depending on the interpretation of $X$ and the choice of $f, f_{i}\left(X_{t}, t\right)$ can be interpreted as, e.g., the power delivered by a power plant or as the instantaneous monetary profit per unit time. Finally, we associate a cost $c_{i j}\left(X_{t}, t\right)$ for switching from production mode $i$ to production mode $j$ at time $t$, where $i, j \in\{1, \ldots m\}$.

The manager of the power plant controls the production by choosing a management strategy, i.e., a combination of a non-decreasing sequence of stopping times $\left\{\tau_{k}\right\}_{k \geq 0}$, where, at time $\tau_{k}$, the manager decides to switch the production from its current mode to another, and a sequence of indicators $\left\{\xi_{k}\right\}_{k \geq 0}$, taking values in $\{1, \ldots, m\}$, indicating the mode to which the production is switched. More precisely, at $\tau_{k}$ the production is switched from mode $\xi_{k-1}$ to $\xi_{k}$ and when starting in mode $i$ at time $t$, we have $\tau_{0}=t$ and $\xi_{0}=i$. We stress that $\tau_{i}$ is required to be a stopping time and as such it is adapted to the filtration $\mathcal{F}^{X}$ generated by the underlying process $X$. In less mathematical terms, this simply means that the decision to switch at time $t$ must be based solely on the information made available up to time $t$, i.e., the manager cannot"peek into the future" when making her decision" 1 .

A strategy $\left(\left\{\tau_{k}\right\}_{k \geq 0},\left\{\xi_{k}\right\}_{k \geq 0}\right)$ can be represented by the random function $\mu:[0, T] \rightarrow\{1, \ldots, m\}$ defined as

$$
\mu_{s} \equiv \mu(s)=\sum_{k \geq 0} \mathbb{q}_{\left[\tau_{k}, \tau_{k+1}\right)}(s) \xi_{k},
$$

which indicates the state of production at time $s$. Given that the stochastic process $X$ starts from $x$ at time $t$, the profit made over $[t, T]$ using a strategy $\mu$ which starts in mode $i$ at time $t$, i.e., a strategy with $\tau_{0}=t$ and $\xi_{0}=i$, is

\footnotetext{
1 Although this is obviously impossible from a practical point of view, it must be stated as an explicit restriction in the mathematical formulation.
} 


$$
J_{i}(x, t, \mu)=E\left[\int_{t}^{T} f_{\mu_{s}}\left(X_{s}, s\right) d s-\sum_{k \geq 1, \tau_{k}<T} c_{\xi_{k-1}, \xi_{k}}\left(X_{\tau_{k}}, \tau_{k}\right) \mid X_{t}=x\right] .
$$

The task of the manager is now to maximize the expected payoff, i.e., to find the most profitable trade-off between switching to more efficient states and minimizing the total cost of switches. In general, this problem, often referred to as an optimal switching problem, consists in finding the value function

$$
u_{i}(x, t)=\sup _{\mu \in \mathcal{A}_{i}(t)} J_{i}(x, t, \mu)
$$

where $\mathcal{A}_{i}(t)$ is a set of strategies with $\tau_{0}=t$ and $\xi_{0}=i$, and the optimal management strategy $\mu^{*} \in \mathcal{A}_{i}(t)$, defined by $\left(\left\{\tau_{k}^{*}\right\}_{k \geq 0},\left\{\xi_{k}^{*}\right\}_{k \geq 0}\right)$, such that

$$
J_{i}\left(x, t, \mu^{*}\right) \geq J_{i}(x, t, \mu)
$$

for any other strategy $\mu \in \mathcal{A}_{i}(t)$.

From the dynamic programming principle, see, e.g., (Tang and Yong 1993), one can derive that the value function $u(x, t)=\left(u_{1}(x, t), \ldots, u_{m}(x, t)\right)$ satisfies the following system of variational inequalities

$$
\begin{aligned}
& \min \left\{-\partial_{t} u_{i}(x, t)-\mathcal{L} u_{i}(x, t)-f_{i}(x, t), u_{i}(x, t)-\max _{j \neq i}\left\{u_{j}(x, t)-c_{i j}(x, t)\right\}\right\}=0, \\
& u_{i}(x, T)=g_{i}(x),
\end{aligned}
$$

for $x \in \mathbb{R}, t \in[0, T], i \in\{1, \ldots, m\}$ and where

$$
\mathcal{L} u(x, t):=\sum_{i=1}^{d_{X}} b_{i}(x, t) \frac{\partial}{\partial x_{i}} u(x, t)+\sum_{i, j=1}^{d_{X}} \frac{1}{2}\left(\sigma \sigma^{*}\right)_{i, j}(x, t) \frac{\partial^{2}}{\partial x_{i} \partial x_{j}} u(x, t)
$$

is the infinitesimal generator of the underlying $d_{X^{-}}$dimensional stochastic process $X$ with dynamics

$$
d X_{t}=b\left(X_{t}, t\right) d t+\sigma\left(X_{t}, t\right) d W_{t}
$$

The operator $\mathcal{L}$ is a linear local operator whenever the process $X$ is driven by a Brownian motion (which we assume in this work) and the system (2.2) has a unique continuous solution under the assumptions of this paper, see, e.g., (Arnarsson et al. 2009; El-Asri and Hamadéne 2009; Lundström et al. 2014b). Moreover, the optimal strategy when starting in mode $i$ at time $t$ is given iteratively by the solution to (2.2) by 


$$
\begin{aligned}
& \tau_{0}^{*}=t, \quad \xi_{0}^{*}=i, \\
& \tau_{k}^{*}=\inf \left\{s>\tau_{k-1}^{*}: u_{\xi_{k-1}^{*}}\left(X_{s}, s\right) \leq \max _{j \neq \xi_{k-1}^{*}}\left\{u_{j}\left(X_{s}, s\right)-c_{\xi_{k-1}^{*} j}\left(X_{s}, s\right)\right\}\right\}, \\
& \xi_{k}^{*}=\underset{j \in\{1, \ldots m\}}{\operatorname{argmax}}\left\{u_{j}\left(X_{\tau_{k}^{*}}, \tau_{k}^{*}\right)-c_{\xi_{k-1}^{*} j}\left(X_{\tau_{k}^{*}}, \tau_{k}^{*}\right)\right\} .
\end{aligned}
$$

\section{Modeling river flow with an SDE}

To apply the theory outlined above to our ROR-hydropower production, we must first find a model for the stochastic flow of water $Q$ following an SDE as in (2.3). More specifically, we are looking for functions $b_{s}$ and $\sigma_{s}$, such that the solution to the SDE

$$
\begin{aligned}
d Q_{t} & =b_{s}\left(Q_{t}, t\right) d t+\sigma_{s}\left(Q_{t}, t\right) d \tilde{W}_{t}, \quad \text { if } t \in[s, T], \\
Q_{s} & =q
\end{aligned}
$$

where $\tilde{W}_{t}$ is a standard Brownian motion, resembles the actual (given) flow of water (in some appropriate sense). This resemblence must hold in the long-term seasonal sense, but at the same time admit for short-term fluctuations due to inter-yearly variations. Our central idea in this regard is to consider the flow of water as the sum of its seasonal variations and a mean-reverting (mean 0) stochastic process, see Sect. 3.1. Forecasts are then treated by altering the estimated seasonal variation on the time-span of the forecast, see Sect. 3.2.

Results indicate that log-transformation of river flow data may increase prediction accuracy of flow models, see (Adnan et al. 2017), and we therefore work with the logarithm of the flow rather than the flow directly.

\subsection{Seasonal variations and short term fluctuations}

Starting with the seasonal variations, we define $R_{t}=\log Q_{t}$ and let $r(t)$ be a function describing the expected value of the logarithm of the flow at time $t$, independent from current observations. Defined as such, $r(t)$ reflects the expected seasonal variation in flow due to spring flood, autumn rains etc., but without any consideration taken to observations from the current year ${ }^{2}$, and we may thus estimate the deterministic function $r(t)$ from historical flow. More precisely, we will construct $r(t)$ as a one-week moving average of the logarithm of the flow, see Sect. 6.1. The choice of a one-week moving average is a trade-off between capturing seasonal variations, such as the spring flood, without letting the mean flow depend too much on the flows of particular years.

\footnotetext{
2 As an example, one would expect that heavy snowfall from January to March would increase the probability of a long and intensive spring flood. Such considerations are not included in $r(t)$.
} 
Next, we consider the fluctuations around the expected mean, $S_{t}=R_{t}-r(t)$, and assume that these fluctuations are given by an Ornstein-Uhlenbeck process reverting towards 0 , i.e.

$$
d S_{t}=-\kappa S_{t} d t+\sigma d W_{t},
$$

where $\kappa>0$ and $\sigma>0$ are constants to be determined and $W_{t}$ is a standard Brownian motion. By standard Itô calculus, the flow $Q_{t}=\exp \left(r(t)+S_{t}\right)$, then satisfies the following stochastic differential equation

$$
d Q_{t}=\left(r^{\prime}(t)+\frac{1}{2} \sigma^{2}-\kappa\left(\log Q_{t}-r(t)\right)\right) Q_{t} d t+\sigma Q_{t} d W_{t},
$$

where $r^{\prime}(t)$ is the derivative of the function $r(t)$. Note that the particular form of (3.1) ensures that $Q_{t}$ stays positive.

With the dynamics (3.1) of the flow in place, what remains is to estimate the parameters $\kappa$ and $\sigma$. To do this, we consider the asymptotic variance and asymptotic autocorrelation for lag $\tau$ of an Ornstein-Uhlenbeck process, which are given by

$$
\text { Variance }=\frac{\sigma^{2}}{2 \kappa} \quad \text { and } \quad \text { Autocorrelation }=e^{-\kappa \tau} \text {, }
$$

repectively. Indeed, for a given set of historical flow data, we take the logarithm of the data and subtract the running-mean $r(t)$ from above to obtain an empirical time series for $S_{t}$. We then calculate the sample autocorrelation function of this time series and estimate the value of $\kappa$ by a linear regression with the logarithm of the sample autocorrelation function as the dependent variable and the lag as the covariate. Finally, we calculate the sample variance of the time series and deduce an estimate of $\sigma$ from the first equation in (3.2) and the estimated value of $\kappa$.

\subsection{Forecasts}

The flow model constructed so far respects seasonal variations whilst still allowing for inter-yearly forecasts. However, in order to perform optimally, our model must also be able to treat short-term fluctuations based on, e.g., weather forecasts or upstream measurements of the flow. Such forecasts will be included in the model by altering the dynamics of $Q$, i.e., by changing (3.1), on a short time span close to the present time $s$.

More precisely, we let for $t \geq s$

$$
d Q_{t}=b_{s}\left(Q_{t}, t\right) d t+\sigma_{s}\left(Q_{t}, t\right) d W_{t},
$$

where

$$
\sigma_{s}(Q, t)=\sigma Q
$$

as before and 


$$
b_{s}(Q, t)= \begin{cases}b_{s}^{f}(Q, t) & \text { if } \quad s \leq t \leq s+l+\ell \\ \left(r_{t}^{\prime}+\frac{1}{2} \sigma^{2}-\kappa\left(\log Q-r_{t}\right)\right) Q & \text { if } \quad s+l+\ell<t\end{cases}
$$

where $b_{s}^{f}$ is a function constructed below. In the above, $l$ and $\ell$ are parameters representing the length of the forecast and the (estimated) time it takes for the forecasted flow to return to the long term mean $r(t)$, i.e., the (estimated) "inertia" of the flow.

Starting at time $s$ with current flow $Q_{s}$ and given a forecast $\left\{F_{r}\right\}_{s<r \leq s+l}$ of the future flow at times $t \in(s, s+l)$ we set

$$
g_{s}(t)= \begin{cases}\log \left(Q_{s}\right) & \text { for } t=s \\ \log \left(F_{t}\right) & \text { for } t \in(s, s+l] \\ \log \left(F_{s+l}\right)+(t-(s+l)) \frac{r_{s+l+\ell}-\log \left(F_{s+l}\right)}{\ell} & \text { for } t \in(s+l, s+l+\ell]\end{cases}
$$

and let

$$
b_{s}^{f}(Q, t)=\left(g_{s}^{\prime}(t)+\frac{1}{2} \sigma^{2}-\kappa\left(\log Q-g_{s}(t)\right)\right) Q .
$$

More explicitly, we calculate the drift $b_{s}^{f}$ as in (3.1) but with the (log-) mean $r(t)$ replaced by $g_{s}(t)$, where $g_{s}(t)$ is given directly by the forecast for $t \in(s, s+l]$, coincides with $r(t)$ for $t>s+l+\ell$ and is linearly interpolated between $s+l$ and $s+l+\ell$. The impact of such forecasts are illustrated in Fig. 3 .

We stress that as time evolves, the forecast will be updated and the function $b_{s}^{f}(t)$ needs to be updated accordingly each time $s$ that a new forecast becomes available. As the starting time $s$ will be clear from context, we will drop the subscript $s$ and simply write $b^{f}(Q, t)$ in the following, although this function varies with $s$ as parameter.

\section{Modeling the payoff structure of power plants}

As mentioned above, we will consider two canonical examples of ROR hydropower plants and outline the payoff structure of these in the current section. We will measure the performance in monetary units (m.u.), but one can easily modify the below to have, e.g., total electricity produced as the trait for optimization.

\subsection{Power plant I: One adjustable unit}

We consider first the simple case of a hydropower plant having a single unit. The unit is designed for the flow $Q_{d}$, but can be run over a wide flow range $\left[Q_{\min }, Q_{\max }\right]$ with lower efficiency. We assume that the unit, automatically and at negligible cost, adjusts to the available flow, and the task of the manager is thus to find out when to start and stop the unit. In the setting of optimal switching, we model the above power plant as follows. The power plant can be run in two states, ' 1 ' and ' 0 ', representing 'on' and 'off', respectively, and for each switch from 0 to 1 or from 1 to 0 the manager must pay a cost 
$c_{01}$ or $c_{10}$, respectively. We assume that the electricity output (in Watts) of the unit when in state 1 is given by

$$
W_{1}(Q)=\left\{\begin{array}{lll}
0 & \text { if } \quad Q<Q_{\min }, \\
c \eta(Q) Q & \text { if } Q_{\min } \leq Q<Q_{\max }, \\
c \eta\left(Q_{\max }\right) Q_{\max } & \text { if } \quad Q_{\max } \leq Q,
\end{array}\right.
$$

where the constant $c$ is simply given by $c=\rho g h$, where $\rho=10^{3} \mathrm{~kg} / \mathrm{m}^{3}$ is the (approximate) density of water, $g=9.82 \mathrm{~m} / \mathrm{s}^{2}$ is the (approximate) gravitational constant, and $h$ is the water head in meters, and $\eta(Q) \in(0,1)$ is the flow-dependent efficiency. In practice, this latter function is given by the specific characteristics of the turbine and generator, but in general it is a concave down function with a maximum at the design flow $Q_{d}$, see, e.g., Fig. 2 and [IPCC (2007), Fig. 9]. To mimic such behaviour, we let

$$
\eta(Q)=\alpha-\beta\left(\frac{Q}{Q_{d}}-1\right)^{2},
$$

where $\alpha$ is the efficiency at design flow and $\beta>0$ quantifies the concavity. Multiplying $W_{1}$ with the spot electricity price and integrating over time gives the income when running the plant in state 1.

To be able to determine an optimal strategy in terms of monetary profit, we need to consider not only the produced electricity, but also running-costs of the plant as well as electricity prices.

We assume that the running cost of the unit is $c_{\text {run }}$ per unit time, the electricity price at time $t$ is $P_{t}$, and that a large additional cost $c_{l o w}$ must be paid for each unit of time that the production unit is run with insufficient flow $Q<Q_{\text {min }}$. The cost $c_{\text {low }}$ is motivated by excessive deterioration of the power plant and the cost should be high enough to avoid running the unit with insufficient flow. Summarizing the above, we find that a reasonable generic payoff function for a unit is given by

$$
f_{1}\left(Q_{t}, P_{t}\right)=-c_{\text {run }}+\left\{\begin{array}{lll}
-c_{\text {low }} & \text { if } & Q_{t}<Q_{\min }, \\
P_{t} c \eta\left(Q_{t}\right) Q_{t} & \text { if } & Q_{\min } \leq Q_{t}<Q_{\max }, \\
P_{t} c \eta\left(Q_{\max }\right) Q_{\max } & \text { if } & Q_{\max } \leq Q_{t},
\end{array}\right.
$$

where $Q_{t}$ and $P_{t}$ represent the flow of water and spot price of electricity at time $t$, respectively, and where the parameters $c, \eta, c_{\text {low }}, c_{\text {run }}, Q_{\min }$ and $Q_{\max }$ in practice are determined by the specific unit under study. We assume that all the above numbers are normalized so that we can take $f_{0} \equiv 0$, i.e., the running cost/payoff when in state 0 is 0 and, without loss of generality, omit fixed costs such as maintenance of buildings, insurances, etc. as these costs do not influence the optimal management strategy. 


\subsection{Power plant II: two homogenous adjustable units}

In our second case, we expand the above power plant with another identical unit so that there are in total three different states available to the manager; running no unit (state 0 ), running a single unit (state 1), or running both units (state 2). As the units are assumed to be identical, there is no difference between running unit 1 or unit 2 in state $2^{3}$. We assume that the payoff structure of both units is as specified in (4.2).

In this setting, a difficulty beyond that of the extra state is introduced. Indeed, with both units running, the manager may control how much of the current flow $Q_{t}$ that is directed towards each of the two running units. With $\delta$ representing the fraction of water directed towards unit 1 , we see that the payoff in state 2 is

$$
f_{1}(\delta Q, P, t)+f_{1}((1-\delta) Q, P, t)
$$

and $\delta$ is thus an additional degree of freedom to optimize over. However, as long as the cost of adjusting units and diverting water is negligible, this optimisation can be done independently of the switching problem. The two-dimensional optimization problem involving two homogenous adjustable units can thus be reduced to a pure switching problem by introducing the payoff function

$$
f_{2}(Q, P, t)=\max _{\delta \in[0,1]}\left\{f_{1}(\delta Q, P, t)+f_{1}((1-\delta) Q, P, t)\right\}
$$

in addition to $f_{0} \equiv 0$ and $f_{1}$ as in (4.2). The optimization in (4.3) can be carried out analytically if $f_{1}$ is sufficiently nice. However, since our final scheme is based on numerical solutions to PDEs, it is merely a question of computational power if the optimization problem (4.3) can only be solved numerically. In any case, this optimization can be done in advance without consideration of current and forecasted flow and to arbitrary precision, making the remaining optimization problem purely a question of optimal switching.

To further facilitate the analysis and understanding of the results, we from here on in let $P_{t} \equiv P_{0}$ and perform our numerical analysis with constant electricity price. However, we stress and that a time-varying deterministic electricity price causes no other problems than obstructing the intuitive understanding of the results.

Remark 1 The above case can easily be expanded to the case of heterogeneous units. In this case, we assume that unit 2 yields payoff

$$
\tilde{f}_{1}(Q, P, t)=-\tilde{c}_{r u n}+\left\{\begin{array}{lll}
-\tilde{c}_{l o w} & \text { if } & Q<\tilde{Q}_{\min }, \\
P c \tilde{\eta}(Q) Q & \text { if } & \tilde{Q}_{\min } \leq Q<\tilde{Q}_{\max }, \\
P c \tilde{\eta}\left(\tilde{Q}_{\max }\right) \tilde{Q}_{\max } & \text { if } & \tilde{Q}_{\max } \leq Q,
\end{array}\right.
$$

\footnotetext{
${ }^{3}$ No difference seen by our model, that is. There could of course be a difference in practice given different wear of the units, planned maintenance, etc.
} 
i.e., unit 2 has the same payoff structure as unit 1 but with parameters

$$
\tilde{Q}_{\text {min }}, \tilde{Q}_{\text {max }}, \tilde{\alpha}, \tilde{\beta}, \tilde{Q}_{d}, \tilde{\eta}, \tilde{c}_{\text {run }} \text {, and } \tilde{c}_{\text {low }} \text {. }
$$

This problem can now be handled in the same way as above by introducing the payoff function

$$
f_{3}(Q, P, t)=\max _{\delta \in[0,1]}\left\{f_{1}(\delta Q, P, t)+\tilde{f}_{1}((1-\delta) Q, P, t)\right\} .
$$

and studying the resulting 4-state optimal switching problem with payoff functions $f_{0}, f_{1}, \tilde{f}_{1}$ and $f_{3}$, depending on whether no, either or both of the units are active.

\subsection{Switching costs}

Allowing for costs when switching between different states is one of the major benefits of an optimal switching approach to production planning. However, these costs (along with the risks involved when switching) are difficult to quantify, and may be so even for an experienced operator. In particular, they depend on the trait of optimization, e.g., maximizing profit, minimizing risk of stoppage, or minimizing wear of the units. Due to the uncertainty regarding the size of these costs, we study the sensitivty of them in Sect. 6, i.e., the performance of our strategy with different (constant) switching costs.

As our purpose is to study the conceptual idea of optimal switching theory in practice (and not to model any particular power plant) we refrain from explicitly modeling switching costs in the current paper. If time- and/or state dependet switching costs $c_{i j}\left(X_{t}, t\right)$ are available, they can be incorporated into the current scheme without any modifications. We encourage further studies on how to model and construct such "switching costs".

\section{Solving the system of PDEs}

With the parameters set above, we are ready to turn to solving (2.2). More precisely, we will solve a discretized version of (2.2) based on the time-discretization $\left\{0=t_{0}, t_{1}, t_{2}, \ldots, t_{N}=T\right\}$ with $\Delta t=t_{n+1}-t_{n}=T / N$, and to do so we utilize an iterative implementation of the Crank-Nicolson scheme outlined in Algorithm 1.

From here on in, we let $\left\{Q_{n} \equiv Q_{t_{n}}\right\}_{0 \leq n \leq N}$ and $\left\{P_{n} \equiv P_{t_{n}}\right\}_{0 \leq n \leq N}$ denote the discrete versions of $Q$ and $P$ based on this discretization. Moreover, starting at time $t_{k}$ with current flow $Q_{k}$, and given a discrete forecast $\left\{F_{t_{k+1}}, \ldots, F_{t_{k+l}}\right\}$ of future flows we define the discrete versions of (3.4) and (3.5) by

$$
\sigma_{k}\left(Q_{n}, t_{n}\right)=\sigma Q_{n}
$$

and 


$$
b_{k}\left(Q_{n}, t_{n}\right)= \begin{cases}b_{k}^{f}\left(Q_{n}, t_{n}\right) & \text { if } \quad k<n \leq k+l+\ell, \\ \left(r^{\prime}\left(t_{n}\right)+\frac{1}{2} \sigma^{2}-\kappa\left(\log Q_{n}-r_{t_{n}}\right)\right) \hat{Q}_{n} & \text { if } \quad k+l+\ell<n,\end{cases}
$$

where

$$
b_{k}^{f}\left(Q_{n}, t_{n}\right)=\left(g_{k}^{\prime}\left(t_{n}\right)+\frac{1}{2} \sigma^{2}-\kappa\left(\log Q_{n}-g_{k}\left(t_{n}\right)\right)\right) Q_{n},
$$

$g_{k}\left(t_{n}\right):=g_{t_{k}}\left(t_{n}\right)$ and $g_{k}^{\prime}$ is calculated as a finite difference $g_{k}^{\prime}\left(t_{n}\right):=\frac{g_{k}\left(t_{n}\right)-g_{k}\left(t_{n-1}\right)}{\Delta t}$.

In the discretized version, and hence in the automated scheme to be constructed, the task is to find a strategy which maximizes the payoff

$$
\sum_{n=0}^{N-1} f_{\mu_{n}}\left(Q_{n}, P_{n}, t_{n}\right) \Delta t-\sum_{N-1 \geq i \geq 0} c_{\mu_{n-1} \mu_{n}}\left(Q_{n}, P_{n}, t_{n}\right)
$$

where $\mu_{n} \equiv \mu\left(t_{n}\right):\left\{t_{0}, t_{1}, \ldots, t_{N}\right\} \rightarrow\{1, \ldots, m\}$ is a discrete valued function indicating the chosen production state between $t_{n}$ and $t_{n+1}$.

Recall that we are aiming at maximizing payoff from the current time $t_{k}$ up to some fixed terminal time $T$. Moreover, the function $b_{k}$ is updated as soon as our forecast is updated (in our case daily) and, consequently, from each starting time $t_{k}$ we will have a new function (5.1) and a new operator $\mathcal{L}^{k}$ in (2.2) (based on the function $b_{k}$ ). Thus, our optimization problem differs slightly from time step to time step and we are bound to solve it repeatedly, for each point $t_{k}$ and with varying time span $\left[t_{k}, T\right]$. For $k$ fixed, our solution procedure is given by pseudo code of Algorithm 1 .

\section{Results}

This section contains results of our parameter estimation and shows the performance of our PDE-based strategy.

\subsection{Parameter values}

We test our model using flow data from the Swedish river Sävarån ${ }^{4}$ during the years 1980-2018, of which we use the first 35 years (1980-2014) for model calibration and the last four (2015-2018) for benchmarking. We also use data from 1980-2014 for a long term evaluation of the performance of our scheme. Leap days are excluded in favour of a coherent presentation of the results.

\footnotetext{
${ }^{4}$ The choice of Sävarån as our data source is made for no other reason than that the river lies close to Umeå University where most of the work on this paper was done.
} 
Result: Numerical approximation of value function $u=\left\{u_{1}, \ldots, u_{m}\right\}$

Initialize $u$ by guessing a solution;

while totalerror $>$ totaltolerance do

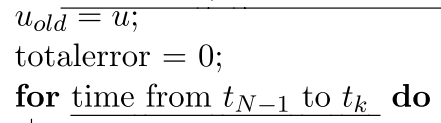

Interpolate linearly at the edges of the spatial grid

end

totalerror $=\left|u_{\text {old }}-u\right|$

end

Algorithm 1: A numerical algorithm for estimating solutions to (2.2).

We study optimization over a one year horizon with possibility to change the state of production once every day, i.e. $T=365$ days and $\Delta t=1$ day $^{5}$.

Remark 2 For hydropower plants with several units, i.e., plant II, the total flow of Sävarån is somewhat low and a non-regulated river with higher flow would be more suitable for our model. For simplicity of the presentation however, we have chosen to limit ourselves to a single river. Specific parameters, such as running costs, deterioration costs, etc., vary over time and between different hydropower plants and the parameters used here should, as with the choice of flow data, be seen merely as an example.

\subsubsection{Flow parameters}

From the historical flow data ${ }^{6}$ of Sävarån, the estimated values of the parameters in (3.2) are $\kappa=0.0208$ and $\sigma=0.1018$. Figure 1a shows $e^{r(t)}$ and the flow during 2015 - 2018 and Fig. 1b shows independent random realisations of (3.1) with these parameters.

\footnotetext{
5 This coarse time-discretization is simply because the flow data available to us is given with one data point per day.

${ }^{6}$ Flow data was downloaded from SMHI (http://vattenwebb.smhi.se/station) on June 15, 2020.
} 


\subsubsection{Power plant parameters}

The parameters used in modeling our power plants are summarized and presented in Table 1.

Starting with the efficiency curve of our power plant units, i.e., the coefficients of (4.1), Fig. 2 shows measured efficiency of two Swedish Kaplan units together with the assumed efficiency curve in (4.1) with least squared fitted coefficients $\alpha$ and $\beta$. Based on this data and the flow in Sävarån, we found it reasonable to choose the unit parameters as in Table 1.

The running cost is estimated from Ström (2012) to be approximately 1/5 of the electricity price. The power of our unit is approximately $500 \mathrm{~kW}$, and with $P_{t}=P_{0} \equiv 1$ it is thus reasonable to set $c_{\text {run }}=100 \mathrm{~m} . u . / \mathrm{h}$. It is difficult to estimate the cost $c_{\text {low }}$ reflecting the running loss when the machine is run on too little water. The rotational speed of the turbine may drop so that the frequency of the produced electricity falls, resulting in a non-sellable production. We choose a value simply by multiplying $c_{\text {run }}$ with 10 and note that such a choice forces our algorithm to efficiently shut down the plant when the water supply fails.

It is not a trivial task to estimate a reasonable value of the switching costs. It may heavily depend on machine parameters related to the intake and specific properties of the turbine, tubes and the generator. Cost of personnel and environmental parameters such as local fish habitat may also be included, as well as the type of contract to which the electricity is sold. Due to these difficulties, we handle the switching costs as a parameter. In particular, we assume the switching costs to be constant and study the impact of varying this constant in Sect. 6.

Moreover, when considering power plant 2, we assume that the cost of switching directly from state 0 to state 2 is cheaper than going through the intermediate state 1 , and vice versa. In particular, this implies that at any fixed time $t$, at most one switch is made. Exactly how much cheaper a direct switch from mode $0 \rightarrow 2$ (or vice versa) should be compared to the alternative $0 \rightarrow 1 \rightarrow 2$ depends on the actual power plant under consideration. Here, we simply assume that the alternative route is $50 \%$ more expensive. We summarize the switching costs in Table 2. Note that we have assumed all switching costs to be positive. When applicable, negative switching costs representing a gain rather than a cost when, e.g., reducing production capacity or moving to a more environmentally friendly production mode, can be used as well.

\subsubsection{Forecasts}

As already stated in the introduction, our main purpose is to highlight the use of optimal switching theory in production planning for ROR hydropower plants. The ambition is not to provide methods for forecasting river flow, and to avoid such discussions, we will simply use the true flow as forecast. However, we keep the stochastic component in the dynamics (3.3) unchanged so that, even with forecast applied, our model does not know the future flow with certainty. Instead, this "forecast" only provides our model with a more accurate estimate of the average flow during the validity period of the forecast. We depict the impact of forecasts in Fig. 3. 


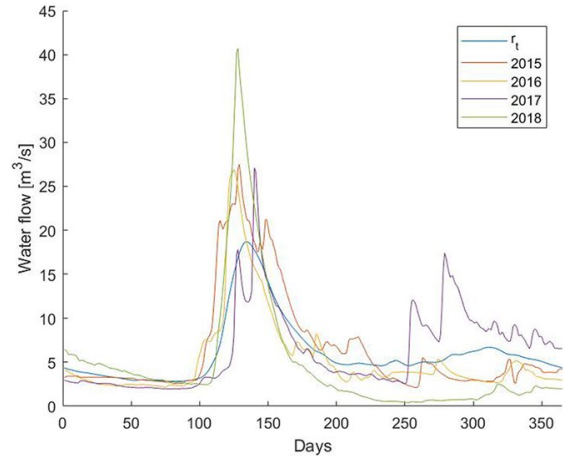

(a) The seasonal model $e^{r(t)}$ together with actual flows (b) The seasonal model $e^{r(t)}$ together with 5 random 2015-2018.

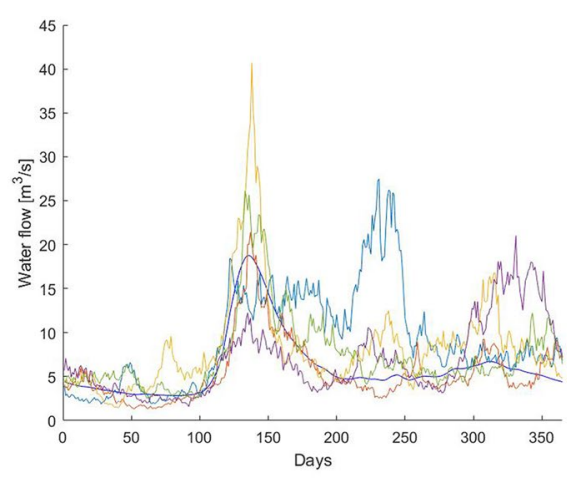

realisations of (3.1).

Fig. 1 A visual comparison of the seasonal model $e^{r(t)}$, solutions to (3.1) and actual flow during 20152018

\subsection{Comparison of different strategies}

We benchmark the performance of our strategy to the a fortiori optimum, i.e., the optimal strategy in hindsight, with all information available. From a practical point of view, this value can only be achieved with certainty by "looking into the future", using the future flow of the river when making decisions. As this is of course impossible in practice, our comparison is slightly skew to the disadvantage of the model presented here. However, this value, the theoretical maximum output of the plant, is indeed achievable and should therefore be considered as the ultimate goal in any attempt to construct a production strategy. We emphasize and stress that, although the benchmark strategy can be found only in hindsight, our PDE-based strategy uses no other information when making a decision at time $t_{k}$ than historical information up to that point and the forecast starting at $t_{k}$.

As a comparison, we also show the result of using a nä̈ve strategy in which the manager always switches to the production mode which momentarily has the highest payoff. To ease the presentation, we assume in all cases that the starting state is 0 , i.e., that the plant is "off" at the beginning of the planning period.

To get comparable results, we normailze with a fixed constant $D$, depending on the power plant under consideration, but not on the switching costs or the flow of the river. More precisely, $D$ is calculated as the profit generated by the plant if it works at maximum capacity for a full year without interruptions (and starting in the most beneficial state), i.e.,

$$
D=f_{i}\left(Q_{\max }, P_{0}, t\right) * 365,
$$

where $i=1$ or $i=2$ depending on the plant under consideration and $t \in[0,365]$ is arbitrary (since (4.2) and (4.3) are independent of $t$ ). After determining $D$, the switching cost constant $C$ (cf. Table 2) is taken as a fixed percentage of $D$.

Last, for comparison of strategies, we use the quotient $\gamma(\mu)$, defined as the total payoff from the strategy $\mu$ divided by $D$, 
Fig. 2 Efficiency values of two Swedish Kaplan units with design flow of approximately $100 \mathrm{~m}^{3} / \mathrm{s}$ (red squares) and $170 \mathrm{~m}^{3} / \mathrm{s}$ (black diamonds). The curves are $\eta(Q)$ defined in (4.1) with least square fitted coefficients. For the smaller unit $\alpha=0.917, \beta=0.430$ (red dotted) and for the larger unit $\alpha=0.935, \beta=0.464$ (black solid)

Table 1 Parameter values used in our numerical investigation

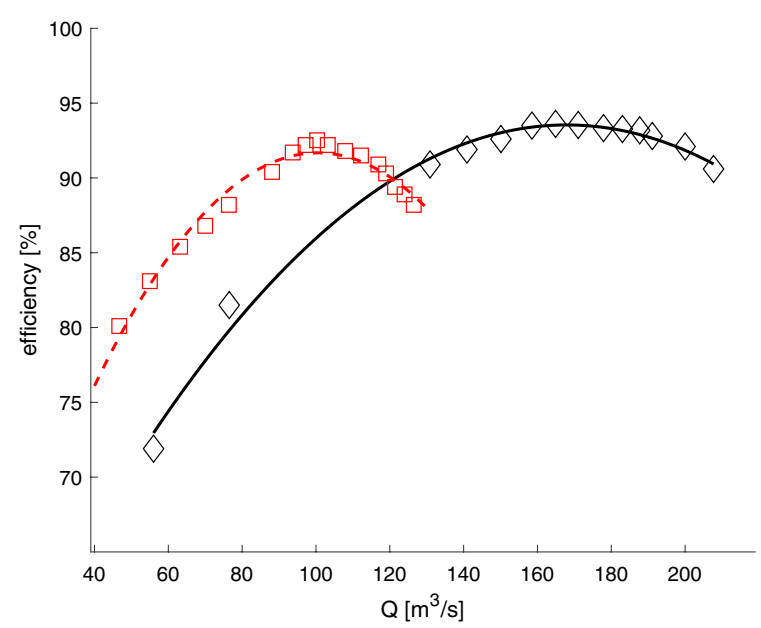

\begin{tabular}{llll}
\hline$P_{0}$ & $1[$ m.u. $/ \mathrm{kWh}]$ & $c_{\text {low }}$ & $1000[$ m.u. $/ \mathrm{h}]$ \\
\hline $\mathrm{h}$ & $5[\mathrm{~m}]$ & $c_{\text {run }}$ & $100[$ m.u. $/ \mathrm{h}]$ \\
$\mathrm{T}$ & $365[$ days $]$ & $Q_{\min }$ & $5\left[\mathrm{~m}^{3} / \mathrm{s}\right]$ \\
$\alpha$ & 0.92 & $Q_{\max }$ & $13\left[\mathrm{~m}^{3} / \mathrm{s}\right]$ \\
$\beta$ & 0.45 & $Q_{d}$ & $10\left[\mathrm{~m}^{3} / \mathrm{s}\right]$ \\
\hline
\end{tabular}

$$
\gamma(\mu)=\frac{J_{0}\left(q_{0}, 0, \mu\right)}{D},
$$

where $q_{0}$ is the flow at the starting time $t_{0}=0$ and $J_{0}$ is as defined in (2.1), i.e., the profit made using strategy $\mu$ over the time horizon $[0, T]$.

Results are provided for $T=365$ days with forecast lengths $l \in\{0,5,10\}$ days and with linear return to the long-term mean $e^{r(t)}$ over $\ell=20$ days. Here, $l=0$ means no forecast. We give detailed descriptions of the suggested strategies for 2015 and show summarized results for $2016-2018$. Moreover, we provide results on the long term performance of our strategy by comparing results from the years $1980-2014$ for a fixed parameter set. ${ }^{7}$

The different production schemes (optimal, PDE-based, naïve) for plant I (II) during 2015, $l \in\{0,5,10\}$ and $C / D=0.01$ are presented in Fig. 5a (Fig. 5b) and Table 3 (Table 4). The relative payoffs as a function of $C / D$ for $l \in\{0,5,10\}$ are

\footnotetext{
${ }^{7}$ It should be noted that, for convenience, this data set is the same as that used for calibrating parameters. Thus, for each year in the long term evaluation, the data tested is part of the data used for calibration. However, a single year out of the 35 used has minimal impact on the end calibration and the long term results are therefore still valid.
} 
Table 2 Relative switching costs. $C$ is a fixed constant determined in Sect. 6

\begin{tabular}{llll}
\hline$c_{i j}$ & $j=0$ & $j=1$ & $j=2$ \\
\hline$i=0$ & 0 & $C$ & $1.5 C$ \\
$i=1$ & $C$ & 0 & $C$ \\
$i=2$ & $1.5 C$ & $C$ & 0 \\
\hline
\end{tabular}

given for all years in Fig. 4 (Fig. 6). The long term performance of the PDE-based strategy for plant I (II) with $C / D=0.01$ is presented in Fig. 7a (Fig. 7b).

The PDE-based strategy in most cases performs very close to the optimal strategy, with a difference of less than $2 \%(5 \%)$ from the theoretical maximum in the long term tests for plant I (plant II) with $C / D=0.01$ and 10 days forecast. The most common mistake of the PDE-based strategy is delaying the decision to change production mode. In all but a few cases, longer forecast also means better results.

\section{Discussion}

The theoretically based optimal swithcing schemes constructed in this paper perform well when tested with real data. When comparing our constructed strategy to the optimal one, we see that differences between these arise as our strategy occasionally recommends switching mode too late due to uncertainty regarding the future flow, see, e.g., Fig. 5b. Most often, the decision is only late by one day and with a finer time discretization these differences would most likely disappear, or at least the discrepancy would be much smaller. We also see that by introducing forecasts, we are able to remove this gap entirely in many cases, see Figs. 4 and 6.

Our (artificial) forecast includes uncertainty from the first forecasted day and reduction in this uncertainty, which is reasonable (and possible by, e.g., upstream measurements), would also help remove delays in the decision making. Indeed, in our SDE model, we assumed the uncertainty to be the same regardless of whether a forecast was introduced or not. If the uncertainty of the forecast is known, one could introduce a new parameter $\sigma_{k}^{f}$ in a similar fashion as for $b_{k}^{f}$ and let the forecast influence also the stochastic volatility of the flow, having lower volatility close to the current time $t_{k}$ and increasing volatility further in the future. We have chosen not to alter the volatility $\sigma$ during the forecast period, partly to keep our model as simple as possible, and partly to avoid the need to construct forecasts (which is outside the scope of the current paper).

Already without forecast, our PDE-based strategy outperforms the naïve strategy in most cases, even for small values of $C / D$, and in many cases also finds the truly optimal strategy or something very close to optimal. In the rare event that the naïve strategy performs as good or better than the PDE-based strategy, it is because the naïve strategy happens to be optimal. In these cases, the difference between the optimal and the PDE-based strategies is small.

Our results show, as should be expected, that longer forecasts give better results. However, on a few occasions, this is not the case, see, e.g., Fig. 4c, where, for 


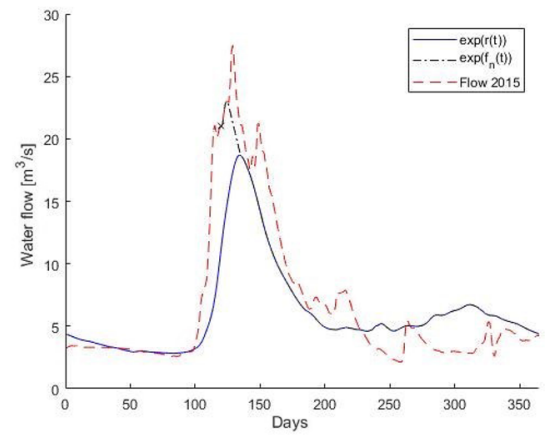

(a) Plot of $e^{r(t)}$, the true flow for year 2015, and $e^{g_{k}(t)}$ for $k=120, l=5$ and $\ell=10$.

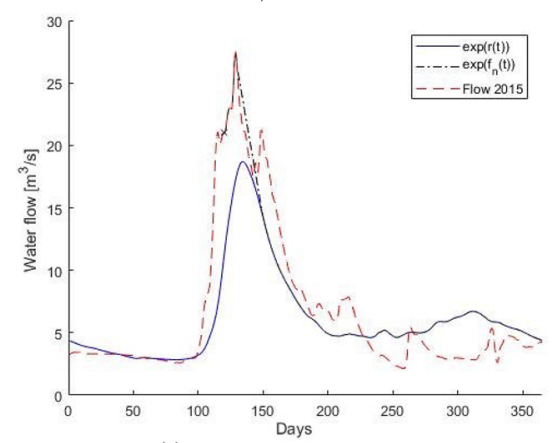

(c) Plot of $e^{r(t)}$, the true flow for year 2015, and $e^{g_{k}(t)}$ for $k=120, l=10$ and $\ell=20$.

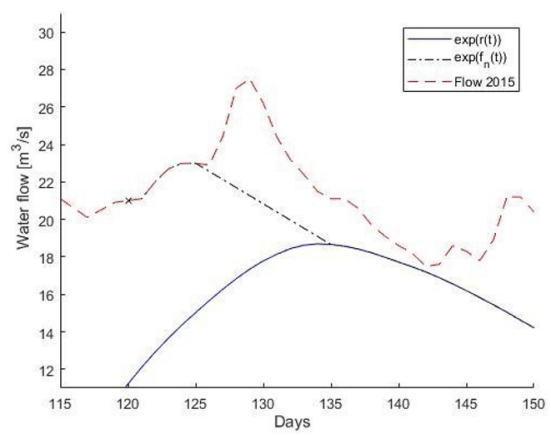

(b) Zoom of figure (a)

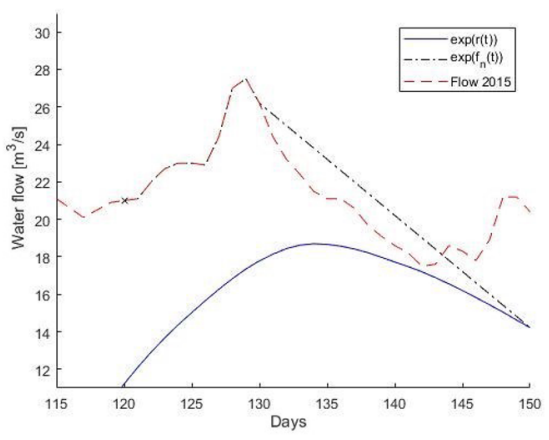

(d) Zoom of figure (c)

Fig. 3 The impact of forecasts in relation to the long-term expected value $r(t)$. Note that a stochastic term is also present in the flow model (3.3) and hence the plotted figure above only corresponds to an estimate of the expected value of the future flow

$C / D \geq 0.016$, we perform worse with a 10 day forecast than with a 5 day forecast. The reason for this is large fluctuations in the flow in just a few days time; the longer forecast then captures both directions of the movement whereas the shorter may only capture one direction. In the latter case, a decision to switch production mode is then made if the flow is close to a level at which different payoff functions intersect. With longer forecast, the uncertainty introduced by forecasting also a rapid downward movement delays the decision slightly when the cost of switching is high. When run repeatedly over a large number of years, the decision made with the longer forecast performs better on average, but it may come up short in a single year. Luckily, as in the comparison with optimal naïve strategies, the deviation in the final payoff is small on these occasions.

Our model is calibrated to a constant electricity spot price $P_{0}$ only for convenience when interpreting results and we repeat that a time-varying deterministic electricity price causes no problems other than parsing and analysing the results. However, our model could also be calibrated to a random price process $P_{t}$ as well. There is no (theoretical) restriction in the number of underlying Markovian Itô processes 
Table 3 Strategies for plant I during 2015 with $C / D=0.01$

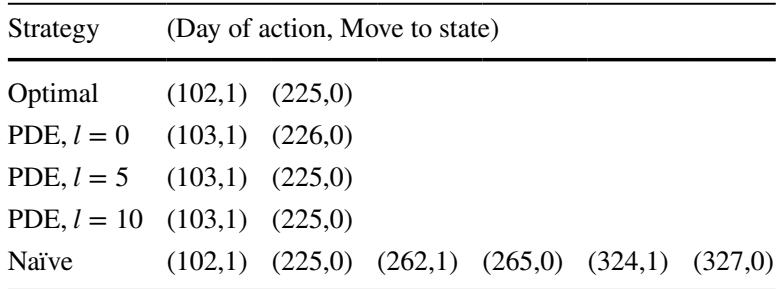

Table 4 Strategies for plant II during 2015 with $C / D=0.01$

\begin{tabular}{|c|c|c|c|c|c|c|c|c|}
\hline \multirow{2}{*}{$\frac{\text { Strategy }}{\text { Optimal }}$} & \multicolumn{8}{|c|}{ (Day of action, Move to state) } \\
\hline & $(102,1)$ & $(111,2)$ & $(159,1)$ & $(225,0)$ & & & & \\
\hline PDE, $l=0$ & $(103,1)$ & $(112,2)$ & $(162,1)$ & $(226,0)$ & & & & \\
\hline PDE, $l=5$ & $(103,1)$ & $(112,2)$ & $(162,1)$ & $(225,0)$ & & & & \\
\hline $\mathrm{PDE}, l=10$ & $(103,1)$ & $(112,2)$ & $(162,1)$ & $(225,0)$ & & & & \\
\hline Naïve & $(102,1)$ & $(111,2)$ & $(159,1)$ & $(225,0)$ & $(262,1)$ & $(265,0)$ & $(324,1)$ & $(327,0)$ \\
\hline
\end{tabular}

our model can handle, so that allowing for such calibration is merely a question of computational power. In fact, not even the Markov property is a restriction as any discretized random process can be made Markovian at the cost of increasing its dimension. However, the cost of increasing the number of random sources is that the underlying optimization problem, which here is solved by PDE-methods, increases in dimensionality at the same rate. In practice, as long as the random sources are few, say 2 or 3, our approach based on numerical solutions of PDE can be used to find a solution. When the dimensionality increases even further, the PDE-methods become computationally heavy and other ways of attacking the resulting optimal switching problem may be preferable, e.g. Monte Carlo-methods as in Aïd et al. (2014), Barkhudaryan et al. (2020). In the current setting, the algorithm for obtaining our strategy is run in only a few minutes on a standard laptop computer and is thus more than sufficiently quick for the purpose of the current paper.

\subsection{Concluding remarks and future research}

In this paper we have, to the authors knowledge for the first time, used the mathematical optimal switching theory to create hydropower production plans which can incorporate random water flow and non-negligible costs of switching between different operational modes. Although our setup is somewhat simplified to keep the analysis of the results tractable, the results are satisfying, showing that automatic optimal switching schemes can perform close to the theoretical maximum already with small computational effort. Moreover, in our study the difference between our model and a naïve approach increased with the number of available production modes, indicating 


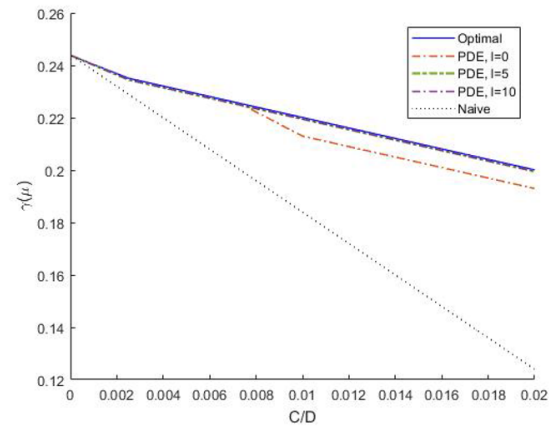

(a) 2015

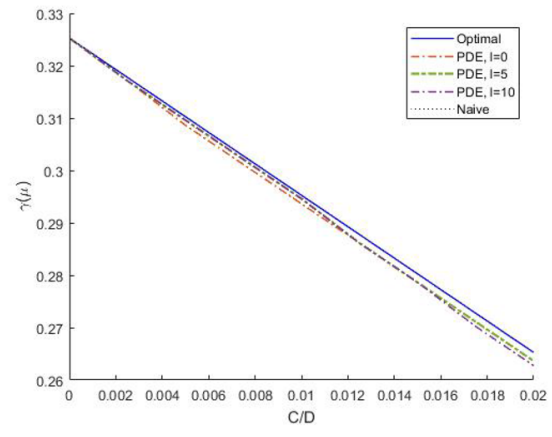

(c) 2017

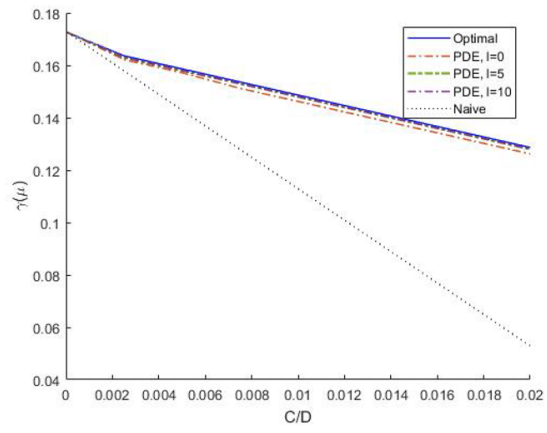

(b) 2016

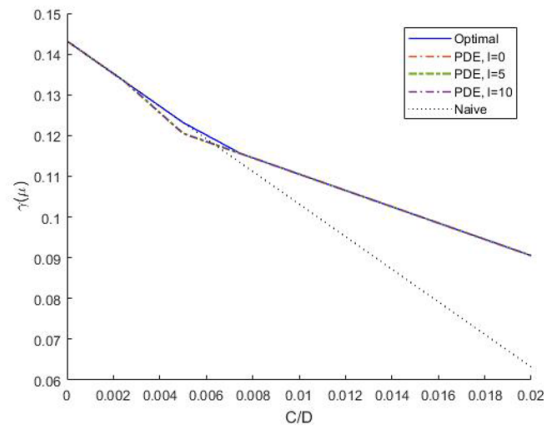

(d) 2018

Fig. 4 Relative payoff for plant I during 2015 - 2018 as a function of $C / D$

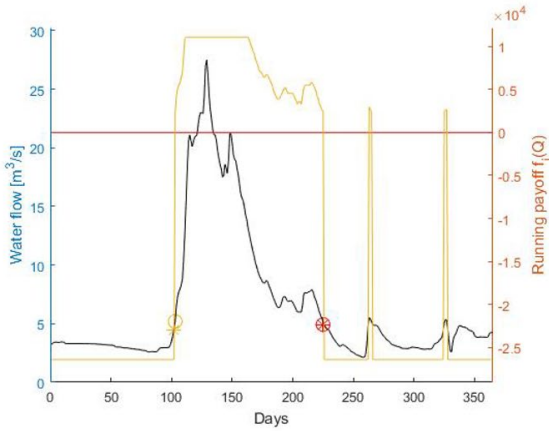

(a) Plant I

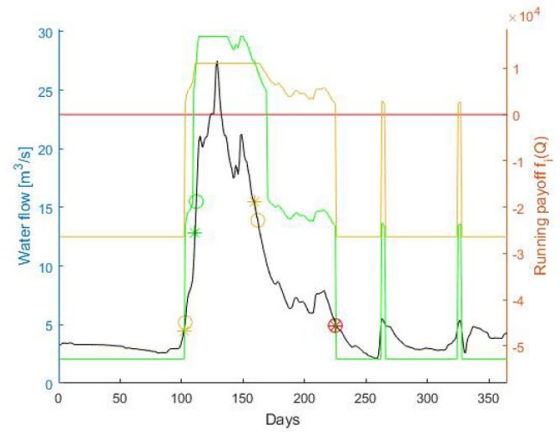

(b) Plant II

Fig. 5 Strategies for plant I (a) and II (b) during 2015 with $C / D=0.01$ and $l=10$. The black curve represents water flow and the red, yellow and green curves represent running payoff for states 0,1 and 2 , respectively. Red, yellow and green circles (asterisks) represent the action of moving to 0,1 or 2 , respectively, under the PDE strategy (optimal strategy) 


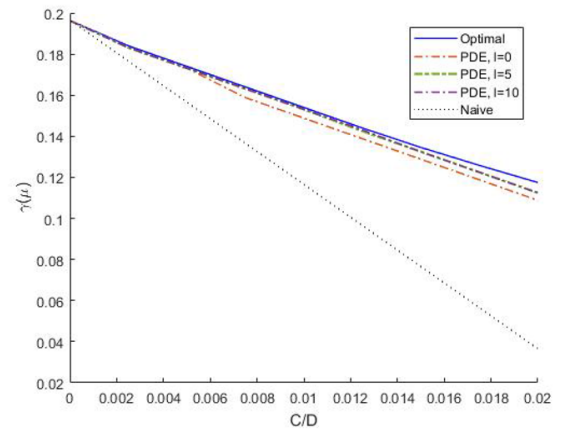

(a) 2015

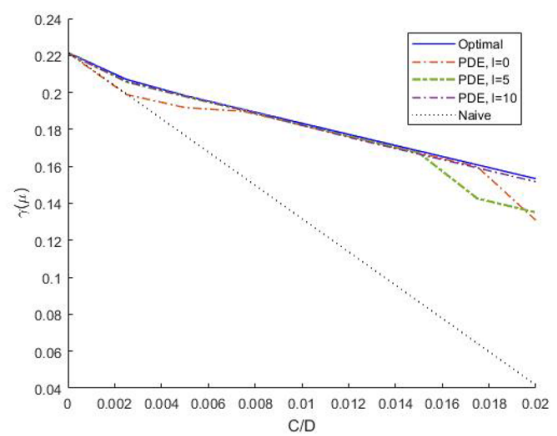

(c) 2017

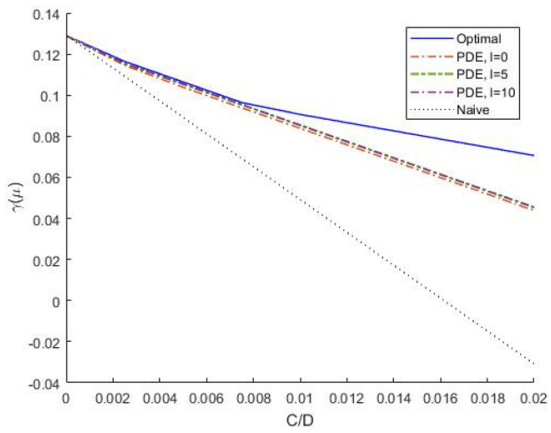

(b) 2016

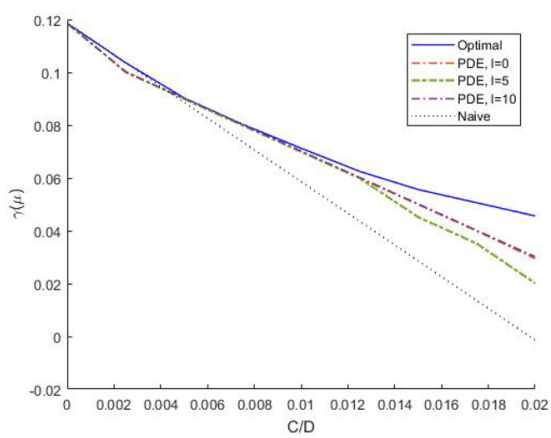

(d) 2018

Fig. 6 Relative payoff for plant II during $2015-2018$ as a function of $C / D$

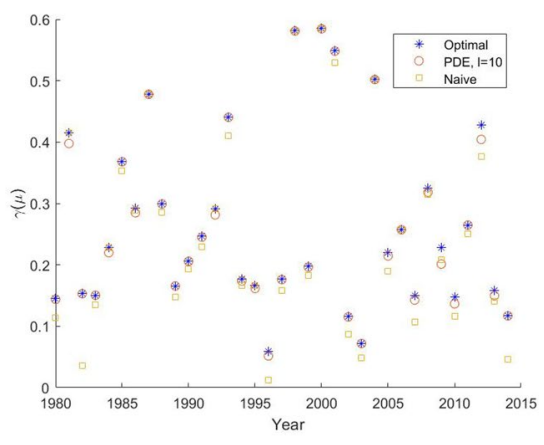

(a) Plant I

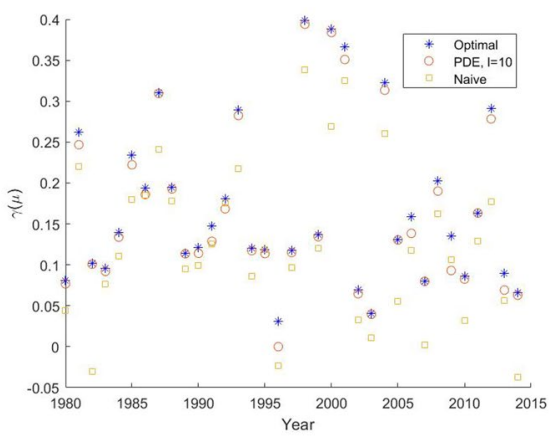

(b) Plant II

Fig. 7 The PDE strategy consistently gives payoff close to the optimal. Here, $C / D=0.01$ and $l=10$. The average quotient $\gamma$ for plant I (II) over the years $1980-2014$ is $0.2674,0.2628$, and $0.2466(0.1708$, 0.1624 , and 0.1244 ) for the optimal strategy, the PDE strategy and the naïve strategy, respectively

that the decision support provided by optimal switching theory becomes increasingly valuable as the complexity of the underlying problem increases. 
An interesting theoretical continuation of the work initiated in this paper would be to study hydropower plants with a dam or hydropower plants of pumped-storage type. At the practitioners' level, a natural next step would be to adapt the current scheme to a real hydropower plant and to benchmark its performance to the production strategy currently in use.

Acknowledgements The work of Niklas L. P. Lundström was partially supported by the Swedish research council grant 2018-03743. Marcus Olofsson gratefully acknowledges the support from the Centre for Interdisciplinary Mathematics at Uppsala University.

Funding Open access funding provided by Umeå University.

Open Access This article is licensed under a Creative Commons Attribution 4.0 International License, which permits use, sharing, adaptation, distribution and reproduction in any medium or format, as long as you give appropriate credit to the original author(s) and the source, provide a link to the Creative Commons licence, and indicate if changes were made. The images or other third party material in this article are included in the article's Creative Commons licence, unless indicated otherwise in a credit line to the material. If material is not included in the article's Creative Commons licence and your intended use is not permitted by statutory regulation or exceeds the permitted use, you will need to obtain permission directly from the copyright holder. To view a copy of this licence, visit http://creativecommons.org/licen ses/by/4.0/.

\section{References}

Adnan RM, Yuan X, Kisi O, Anam R (2017) Improving accuracy of river flow forecasting using LSSVR with gravitational search algorithm. Adv Meteorol. Article ID: 2391621

Aïd R, Campi L, Langrené N, Pham H (2014) A probabilistic numerical method for optimal multiple switching problems in high dimension. J Fin Math 5:191-231

Anagnostopoulos JS, Papantonis DE (2007) Optimal sizing of a run-of-river small hydropower plant. Energy Convers Manag 48(10):2663-2670

Arnarsson T, Djehiche B, Poghosyan M, Shahgholian H (2009) A PDE approach to regularity of solutions to finite horizon optimal switching problems. Nonlinear Anal 71(12):6054-6067

Barkhudaryan R, Gomes DA, Shahgholian HM (2020) System of variational inequalities with interconnected obstacles. Appl Anal. https://doi.org/10.1080/00036811.2020.1757076

Biswas IH, Jakobsen ER, Karlsen KH (2010) Viscosity solutions for a system of integro-PDEs and connections to optimal switching and control of jump-diffusion processes. Appl Math Optim 62:47-80

Bodo BA, Thompson ME, Unny TE (1987) A review on stochastic differential equations for applications in hydrology. Stoc Hydrol Hydraul 1:81-100

Boldea I (2015) Synchronous generators. CRC Press, Boca Raton

Bozorg Haddad O, Moradi-Jalal M, Mariño MA (2011) Design-operation optimisation of run-of-river power plants. Proc Inst Civ Eng Water Manag 164(9):463-475

Brekke KA, Øksendal B (1994) Optimal switching in an economic activity under uncertainty. SIAM J Control Optim 32:1021-1036

Brekke H (2001) Hydraulic turbines design, erection and operation. Norwegian University of Science and Technology (NTNU) publications, Norway

Brennan MJ, Schwartz ES (1985) Evaluating natural resource investments. J Bus 58(2):135-157

Carmona R, Ludkovski M (2008) Pricing asset scheduling flexibility using optimal switching. Appl Math Fin 15:405-447

Carmona R, Ludkovski M (2010) Valuation of energy storage: An optimal switching approach. Quan Fin 10:359-374

Djehiche B, Hamadéne S, Popier A (2010) A finite horizon optimal multiple switching problem. SIAM J Control Optim 48:2751-2770 
Duckworth K, Zervos M (2001) A model for investment decisions with switching costs. Ann Appl Prob 11(1):239-260

El-Asri B, Fakhouri I (2017) Viscosity solutions for a system of PDEs and optimal switching. IMA J Math Control Inform 34(3):937-960

El-Asri B, Hamadéne S (2009) The finite horizon optimal multi-modes switching problem: the viscosity solution approach. Appl Math Optim 60:213-225

Fahlbusch F (1983) Optimum capacity of a run-of-river plant. Int Water Power Dam Constr 35(3):45-48

Fathian F, Fard AF, Ouarda TBMJ, Dinpashoh Y, Nadoushani SSM (2019) Modeling streamflow time series using nonlinear SETAR-GARCH models. J Hydrol 573:82-97

Garrido J, Zafra A, Vazquez F (2009) Object oriented modelling and simulation of hydropower plants with run-of-river scheme: a new simulation tool. Simu Model Pract Theory 17(10):1748-1767

Goutte S, Kharroubi I, Lim T (2018) Optimal management of an oil exploitation. Int J Global Energy Issues 41:69-85

Hamadéne S, Morlais MA (2013) Viscosity solutions of systems of PDEs with interconnected obstacles and switching problem. Appl Math Optim 67:163-196

Hellström J, Lundgren J, Yu H (2012) Why do electricity prices jump? Empirical evidence from the Nordic electricity market. Energy Econ 34:1774-1781

$\mathrm{Hu}$ Y, Tang S (2010) Multi-dimensional BSDE with oblique reflection and optimal switching. Prob Theory Relat Fields 147:89-121

IPCC, Renewable, (2007) Energy sources and climate change mitigation: special report of the intergovernmental panel on climate change-chapter 5, Hydropower. Cambridge University Press: New York, USA

Kharroubi I (2016) Optimal switching in finite horizon under state constraints. SIAM J Control Optim 54:2202-2233

Lundström NLP, Nyström K, Olofsson M (2014) Systems of variational inequlities for nonlocal operators related to optimal switching problems: existence and uniqueness. Manus Math 145:407-432

Lundström NLP, Nyström K, Olofsson M (2014) Systems of variational inequalities in the context of optimal switching problems and operators of Kolmogorov type. Ann di Math Pura ed Appl 193:1213-1247

Lundström NLP, Olofsson M (2021) Systems of fully nonlinear parabolic obstacle problems with Neumann boundary conditions. Preprint, arXiv:2105.01983

Lundström NLP, Olofsson M, Önskog T (2019) Existence, uniqueness and regularity of solutions to systems of nonlocal obstacle problems related to optimal switching. J Math Anal Appl 475(1):13-31

Martyr R (2016) Dynamic programming for discrete-time finite-horizon optimal switching problems with negative switching costs. Adv Appl Prob 48:832-847

Martyr R (2016) Finite-horizon optimal multiple switching with signed switching costs. Math Op Res 41:1432-1447

Modarres R, Ouarda TBMJ (2013) Modelling heteroscedasticity of streamflow time series. Hydrol Sci J 58(1):54-64

Moeeni H, Bonakdari H, Fatemi SE, Zaji AH (2017) Assessment of stochastic models and a hybrid artificial neural network-genetic algorithm method in forecasting monthly reservoir inflow. INAE Lett 2(1):13-23

Mujumdar PP, Nagesh Kumar D (1990) Stochastic models of streamflow: some case studies. Hydrol Sci J 35(4):395-410

Matos JP, Schleiss AJ (2017) A free and state-of-the-art probabilistic flow forecasting tool designed for Africa, In: Proceedings of the water storage and hydropower development for Africa conference

Paish O (2002) Small hydro power: technology and current status. Renew Sustain Energy Rev 6(6):537-556

Perninge M (2018) A limited-feedback approximation scheme for optimal switching problems with execution delays. Math Methods Op Res 87(3):347-382

Perninge M (2020) A finite horizon optimal switching problem with memory and application to controlled SDDEs. Math Methods Op Res 91(3):465-500

Perninge M (2020) On the finite horizon optimal switching problem with random lag. Appl Math Optim. https://doi.org/10.1007/s00245-019-09648-0

Perninge M (2020) A two-scale scheme for finite horizon switching problems with delays. Automatica 112:108709

Perninge M, Eriksson R (2017) Frequency control in power systems based on a regulating market. IEEE Trans Control Syst Technol 26(1):27-37 
Ström M (2012) Småskalig vattenkraft: Dagsläge och framtidsplaner. Examensarbete INDEK, KTH 2012:100

Tang SJ, Yong JM (1993) Finite horizon stochastic optimal switchingand impulse controls with a viscosity solution approach. Stoc Stoc Rep 45:145-176

Wang W, Van Gelder PHAJM, Vrijling JK, Ma J (2005) Testing and modeling autogregressive conditional heteroskedasticity of streamflow processes. Nonlinear Process Geophys 12:55-66

Warnick CC (1984) Hydropower engineering. Prentice-Hall, U.S

Weron R, Bierbrauer M, Truck S (2004) Modeling electricity prices: jump diffusion and regime switching. Phys A Stat Mech Appl 336:39-48

Weron R, Simonsen I, Wilman P (2004) Modeling highly volatile and seasonal markets: evidence from the Nord Pool electricity market. In: The application of econophysics. Springer: Tokyo, pp 182-191

Yang J (2010) Underwater tunnel piercing in refurbishment of Akkats power station, ICOLD Annual Meeting and Symposium, May 2010, Hanoi, Vietnam

Yang J, Andreasson P, Högström C-M, Teng P (2018) The tale of an intake vortex and its mitigation countermeasure: a case study from akkats hydropower station. Water 10(7):881

Publisher's Note Springer Nature remains neutral with regard to jurisdictional claims in published maps and institutional affiliations. 